\title{
PROPUESTAS CURRICULARES PARA EL PLAN DE ESTUDIOS DEL AÑO 2000 DE LA FACULTAD DE CIENCIAS CONTABLES DE LA UNMSM
}

Dr. ERNESTO A. POLAR FALCÓN

\section{INTRODUCCIÓN}

En 1996, las autoridades de la Facultad de Ciencias Contables de la UNMSM, tomaron la decisión de modificar sustancialmente el Plan de Estudios que se aplicaría en dicho año académico. El objetivo era formar un Contador Público acorde con el avance de la ciencia contable y con los requerimientos del mercado empresarial moderno. Las promociones de Egresados cada año, desde 1996 a 1999, han asimilado en mayor proporción los nuevos enfoques y conceptos del nuevo Plan de Estudios 1996; y la Promoción de Egresados 2000 que saldrá en diciembre de 2000 , será la primera formada íntegramente con el vigente y moderno Plan de Estudios 1996.

Los resultados han sido satisfactorios para los alumnos de la Facultad de Ciencias Contables de la UNMSM, por cuanto, desde 1996 a la fecha, la demanda de asistentes y auxiliares de contabilidad por parte de las empresas, ha subido notoriamente. Este hecho se comprueba con la gran cantidad de cartas que mensualmente recibe la Facultad provenientes de empresas privadas e instituciones públicas; así como el interés de entidades del Estado y del sector financiero de solicitar la participación de estudiantes en la selección por concurso, para brindar entrenamiento previoy posterior contrato de trabajo.

También los Contadores Públicos egresados de las indicadas promociones, con sus flamantes títulos profesionales, han sido seleccionados para ocupar importantes cargos de responsabilidad, después de rigurosas selecciones, en un marco de alta competitividad.

Estas son las razones que impulsan a mantener un constante estudio de avance curricular en la formación del Contador Público, como tarea propia de la investigación metodológica, que propicia el Instituto de Investigaciones de Ciencias Financieras y Contables, como miembro del CSI (Consejo Superior de Investigaciones) de la UNMSM; que en su Boletín 30 (marzo 1999), define a la investigación como "la actividad intelectual y creativa que procura describir nuevos hechos, evaluándolos $\mathrm{e}$ interpretándolos a la luz de conocimientos previos. Esta actividad, ya sea que busque ampliar conocimientos por sí misma u obtener resultados de valor específico, económico y social, su razón de ser es su contribución al bienestar humano".

Asimismo, en la Revista InterAmérica de la Asociación Interamericana de Contabilidad (julio - setiembre 1998), en el artículo "Reflexiones en torno a la relación docencia-investigación en materia contable" de John Cardona Arteaga, dice que: "Es moneda corriente afirmar que la universidad no sólo ha de limitarse a transmitir los conocimientos existentes de las diferentes disciplinas científicas o técnicas (docencia), si no que también ha de buscar nuevos conocimientos que aumenten o perfeccionen 
el acervo de los acumulados hasta el momento de las distintas disciplinas, con el fin de conseguir nuevos progresos científicos o técnicos que produzcan o impliquen un impacto más favorable para el hombre en su comprensión y utilización de la naturaleza (investigación). Docencia e investigación constituyen, en consecuencia, un binomio inseparable del quehacer universitario, cualquiera que sea el área de conocimiento, siendo ambos imperativos insoslayables para los profesores universitarios".

$\mathrm{Y}$ en base a lo expuesto, cabe preguntar: ¿Por qué es necesario mantener el interés en los estudios de investigación relacionados con proponer cambios curriculares en la formación del Contador Público en el Perú, como noble tarea educativa y académica de la Facultad de Ciencias Contables de la UNMSM?

Una de las respuestas a tal pregunta está en la Revista InterAmérica de la Asociación Interamericana de Contabilidad (octubrediciembre 1998), en el artículo "El Contador Público hacia el Próximo Milenio", que textualmente dice: "Desde Lucas Paccioli, la base de la contabilidad es la misma. Es tan simple que no ha sido necesario cambiarla. Sin embargo, es el mundo el que no es el mismo, ¿por qué?:

a) El desarrollo del comercio internacional ha extendido las fronteras y con ello ha llevado los aspectos a considerar a ámbitos, legislaciones y prácticas muchas veces no muy familiares.

b) La aparición de nuevos instrumentos financieros. En efecto, casi a diario se "crean" nuevos y, a veces complejas herramientas que nos obligan a meditar cuidadosamente su influencia contable.

c) La nueva concepción empresarial, donde se privilegian los "flujos" y se va a mundo de grandes fusiones, aún ello ocurre en nuestra propia profesión, derivada de la necesidad de atender esos enormes mercados que se están abriendo, con millones de habitantes. Esto nos obliga a revisar muchos conceptos, arraigados en algunos de nuestros países, sobre si podemos o no asociarnos con otras profesiones. Un caso especial es el de la asociación con abogados, por el tema tan delicado de los conflictos de intereses.

d) El formidable avance de las comunicaciones. En efecto, tanto en nuestras propias tareas como en las relaciones de clientes que operan en muy diferentes latitudes, las nuevas concepciones técnicas de las comunicaciones, nos obliga a un estudio cuidadoso para que ello signifique un aspecto positivo de nuestros servicios profesionales y no, por el contrario, un obstáculo que desmerezca nuestro valor agregado.

Y otra respuesta se plantea en el último "III Foro de la Universidad Peruana", realizado del 21 al 23 de octubre de 1999 en la ciudad de Trujillo, bajo el lema de "Gobierno, Empresa y Universidad: Por el Desarrollo", que en el Tema IV: "La Universidad, su desarrollo y el desarrollo del país", plantean siete inquietudes; las principales son:

- ¿La Universidad y sus funciones principales están acordes con la realidad actual? ¿Basta tener liderazgo académico, formas profesionales, dar formación técnica y de perfeccionamiento o es necesario darle presencia acorde con la realidad y las propuestas de desarrollo del país?

- ¿Qué tipo de iniciativas y formas de acción son indispensables para que se entienda que lo que viene trabajando la universidad está vinculada a 
la construcción de sociedades más productivas, informadas, prósperas, justas, satisfactorias y democráticas, que sin lugar a dudas forman parte esencial de la propuesta de desarrollo?

\section{LOS OBJETIVOS DEL ESTUDIO}

Al inicio del presente estudio se fijaron los dos siguientes objetivos:

1. Analizar los avances curriculares en la formación profesional del Contador Público en México, que recibe de Norteamérica información contable actualizada; países que poseen gran capacidad de traducción al idioma español. Se programarán estas actividades dentro de los parámetros académicos de los convenios firmados entre la UNMSM y las universidades latinoamericanas.

2.Estudiar los contenidos curriculares de los cursos de reciente implantación en México y Argentina, para establecer su adecuación al Perú y su incorporación al Plan de estudios de la Facultad de Ciencias Contables de la UNMSM.

\section{LIMITACIONES PRESENTADAS EN LA INVESTIGACIÓN}

Por razones de austeridad no se concretaron las visitas programadas, tanto a la Facultad de Contaduría y Administración de la Universidad Nacional Autónoma de México, como a la Facultad de Ciencias Económicas de Universidad de Buenos Aires-Argentina.

Ambas visitas de trabajo tenían como objetivos específicos, recolectar informaciones curriculares, que comprendían desde conocer el vigente plan curricular de estudios, con sus componentes de perfil académico y profesional del futuro Contador Público, del Plan de Estudios que abarcan los cinco años de estudios, del contenido de las áreas de estudio, sumillas y Syllabus de cada curso, hasta la evaluación curricular para analizar los resultados obtenidos al finalizar la carrera de estudios y la aceptación profesional en el marco de las ofertas de trabajo que ofrecen las empresas en ambos países.

Además, se tenía programado revisar la bibliografía relacionada los avances curriculares en otras universidades de ambos países y en las universidades latinoamericanas y del mundo, principalmente de Europa.

\section{EL PROCESO DE INVESTIGACIÓN APLICADO}

El presente estudio se ha realizado en las cinco siguientes fases:

\section{Planeación:}

En los formularios proporcionados por el Consejo Superior de Investigaciones de la UNMSM, se presentó oportunamente el plan de acción que se iba a aplicar durante el trabajo inquisitivo.

\section{Recopilación:}

- Se ha obtenido por vía Internet, el siguiente material de trabajo:

\section{UNIVERSIDAD NACIONAL AUTÓ- NOMADEMÉXICO}

Facultad de Contaduría y Administración

Licenciado en Contaduría

Plan de Estudios

UNIVERSIDAD DE BUENOS AIRES ARGENTINA

Facultad de Ciencias Económicas

Contador Público

Plan de Estudios 
- Se ha obtenido por intercambio curricular, el siguiente material de trabajo:

UNIVERSIDAD AUTÓNOMA DE GUADALAJARA-MÉXICO

Contador Público

UNIVERSIDAD CATÓLICA DE CÓRDOVA-ARGENTINA

Contador Público

UNIVERSIDAD AUTÓNOMA DE COLOMBIA

Contador Público

- Se ha obtenido de la XXIII Conferencia Interamericana de Contabilidad, Puerto Rico, del 2 al 5 de agosto de 1999, el siguiente material de trabajo:

TRABAJOS INTERAMERICANOS:

(En la Biblioteca de la Facultad de Ciencias Contables).

•Área 4 - EDUCACIÓN

Currículos para el profesional de Clase Mundial:

"Currículos para un profesional de Clase Mundial" - Yvonne L. Huertas

Interpretación de Enfoque Interdisciplinarios al Currículo:

"Interdisciplinariedad en la Formación Integral del Contador Público".

TRABAJOS NACIONALES:

(En la Biblioteca de la Facultad de Ciencias Contables).

\section{•Área 4 - EDUCACIÓN}

Currículos para el profesional de Clase Mundial:

"Evaluación preliminar de las Posibilidades de Integración de la Tecnología Multimedia en los Procesos de Formación de Contadores Públicos" - Elsa B. Suárez Kimura.

"Retos para la Formación del Contador Público de Clase Mundial" - Osvaldo A.Cháves - Ricardo Pahlen Acuña - Juan Carlos Da Silva.

Integración de Enfoques Interdisciplinarios al Currículo:

“Necesidades de Investigar Formas y Procedimientos de Investigación Curricular en la Carrera de Contador Público"' - Mario Biondi.

"El Contador: Pensador Estratégico y Forjador del Fututo" - Horacio López Santiso.

"Lo Interdisciplinario y la Formación Profesional en el Siglo XXI: El Currículum del Contador" - Sebastián Ferril Márquez.

\section{Procesamiento:}

En esta fase del estudio, se analizaron los Planes de Estudios obtenidos de la Facultad de Contaduría y Administración de la Universidad Nacional Autónoma de Méxicoy de la Facultad de Ciencias Económicas de la Universidad de Buenos Aires Argentina.

Específicamente, se revisaron los contenidos de cada curso y se ordenaron los cursos por áreas, temas o líneas de estudios. 


\section{Interpretación:}

Para explicar el contenido de la información obtenida, se ha usado la siguiente metodología:

a) La lectura de la sumilla de cada curso.

b) En base a la lectura efectuada se han agrupado los cursos por su afinidad académica en catorce (14) temas como sigue:

1. Contabilidad

2. Costos

3. Finanzas

4. Economía

5. Administración

6. Matemáticas

7. Informática

8. Comunicación

9. Inglés

10. Tributación

11. Derecho

12. Auditoría

13. Estadística

14. Humanidades

c) Se ha colocado en una gráfica, los catorce (14) temas y se ha identificado con símbolos a tres (3) universidades, agregando el cronograma de estudios que aplican en los cinco (5) años académicos, tal como se indica a continuación:

UNMSM - Universidad Nacional Mayor de San Marcos, Lima - Perú Símbolo (-.-)

Cronograma de estudios: - 5 Años Académicos

UBA - Universidad de Buenos Aires - Argentina

Símbolo (...)

Cronograma de estudios:
- $1^{\circ}$ Tramo

Ciclo General

- $2^{\circ}$ Tramo

Ciclo General

- $1^{\circ}$ Tramo

Ciclo Profesional

- $2^{\circ}$ Tramo

Ciclo Profesional

UNAM - Universidad Nacional Autónoma de México

Símbolo (---)

Cronograma de estudios:

- 10 Semestres Académicos

d) Se le ha puesto el siguiente título a la gráfica:

ANÁLISIS COMPARATIVO DE LOS PLANES DE ESTUDIOS DE TRES UNIVERSIDADES (Ver Figura A-1).

e)Con líneas horizontales, se ha procedido a anotar los cursos que por afinidad académica, corresponden a un tema específico, de los catorce (14) nombrados. 
FIGURA A - 1

\section{ANÁLISIS COMPARATIVO DE LOS PLANES DE ESTUDIOS} DE TRES UNIVERSIDADES

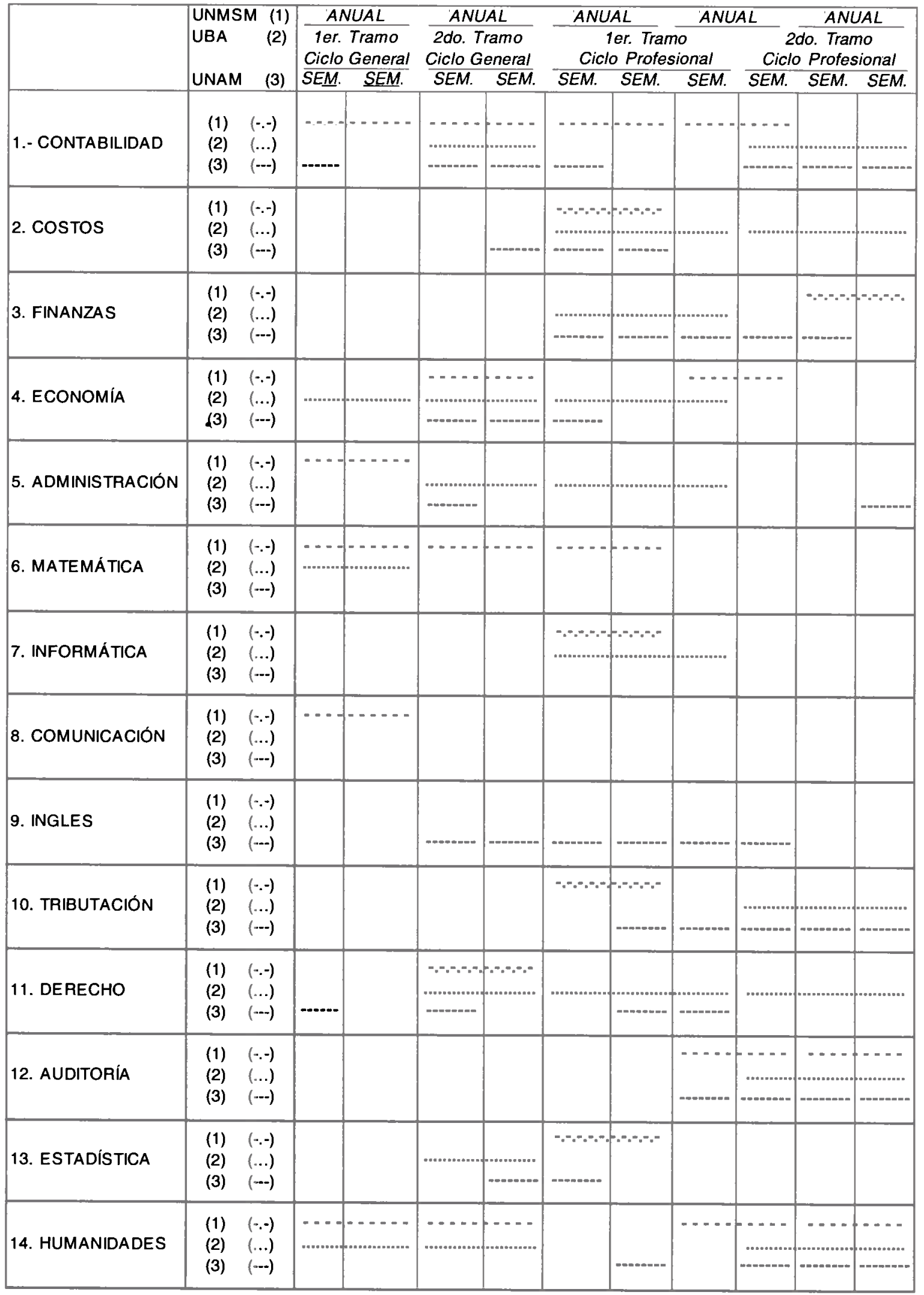


A continuación se explica en el tema: "1. Contabilidad" la forma de interpretar y comparar los cursos de cada universidad:

UNMSM (1) - Símbolo (-.-)

Se dictan cursos de Contabilidad en:

- Primer Año

- Segundo Año

- Tercer Año

- Cuarto Año

UBA (2) - Símbolo (...)

Se dictan cursos de Contabilidad en:

- $2^{\circ}$ Tramo - Ciclo Profesional

(La doble línea horizontal significa dos cursos del tema en este tramo)

UNAM (3) - Símbolo (---)

Se dictan cursos de Contabilidad en:

- Primer Semestre

- Segundo Semestre

- Tercer Semestre

- Cuarto Semestre

(Dos cursos del tema)

- Quinto Semestre

- Octavo Semestre

- Noveno Semestre

- Décimo Semestre

\section{Comunicación:}

En esta última fase del proceso de investigación, se presentan los resultados obtenidos, en beneficio del Plan de Estudios de la Facultad de Ciencias Contables de la UNMSM, como sigue:

1. Contabilidad: En la UNAM se aprecia con interés el contenido de los tres (3) siguientes cursos:

\section{- Octavo Semestre: Contabilidades Especializadas}

Técnicas contables de acuerdo a los sectores económicos, sus generalidades, su régimen jurídico y fiscal, su sistema contable y sus requerimientos de información.
-Noveno Semestre: Contabilidad Internacional

Los efectos contables que se presentan en las relaciones comerciales internacionales.

\section{- Décimo Semestre: Seminario de Contabilidad}

Casos representativos de problemas generales de la contaduría y propuesta de soluciones a dichos casos.

\section{Costos:}

En la UBA se aprecia con interés el contenido de los dos (2) siguientes cursos:

\section{- $1^{0}$ Tramo - Ciclo Profesional: Gestión y Costos}

Teoría General del costo. Modelos. La Gestión y el control de los costos. Productividad, eficacia y eficiencia. Planificación y Presupuestos; control. Decisiones de corto y largo plazo en base a los costos. Sistemas de equilibrio e indiferencia. Los costos y las técnicas de gestión. Reducción de Costos. Proceso gerencial e información contables y extracontables.

\section{-2 Tramo - Ciclo Profesional:} Sistemas de Costos

Factores y componentes del costo. Técnicas y métodos de determinación De costos. Costeo de productos, funciones y actividades, históricos y predeterminados. Ciclo Contable del costo y el resultado. Estados e informes de costos y resultados. Control contable de costos y presupuestos. Evaluación de actuación a través de los costos y los resultados.

\section{Informática:}

En la UNAM se aprecia con interés el contenido de los cinco (5) siguientes cursos: 
-Primer Semestre: Informática I

Estructura, funciones, capacidades y limitaciones de una computadora aplicada a su campo profesional. Dominio del sistema operativo, del procesador de textos. Análisis de la conceptualización y campo profesional actual del Licenciado en Informática Administrativa.

-Segundo Semestre: Informática II

Dominio de la hoja de cálculo y del graficador.

-Tercer Semestre: Informática III Dominio del sistema de base de datos.

-Cuarto Semestre: Informática IV Dominio de los sistemas administrativos y del sistema contable.

- Quinto Semestre: Informática V Dominio del sistema de edición e impresión y perspectivas de desarrollo informático.

\section{Comunicación:}

En la UNAM se aprecia con interés el contenido de los dos (2) siguientes cursos:

-Primer Semestre:Comunicación I El proceso de comunicación escrita; técnicas para mejorar la expresión escrita; aplicación de dichas técnicas a casos prácticos.

-Segundo Semestre: Comunicación II. El proceso de comunicación no escrita, técnicas para mejorar la expresión no escrita y su aplicación a casos prácticos.

\section{Inglés:}

En la UNAM se aprecia con interés el contenido de los seis (6) siguientes cursos:

\section{-Tercer Semestre: Inglés I}

Expresiones de uso en inglés, a nivel de las cuatro habilidades básicas: hablar, leer, entender y escribir; reglas de la lengua inglesa, según su aplicación en textos escritos. Comprensión de textos técnicos, científicos y literarios escritos en inglés.

\section{-Cuarto Semestre: Inglés II}

(Idem al Tercer Semestre)

-Quinto Semestre: Inglés III

(Idem al Tercer Semestre)

-Sexto Semestre: Inglés IV

(Idem al Tercer Semestre)

-Séptimo Semestre: Inglés V

(Idem al Tercer Semestre)

-Octavo Semestre: Inglés Técnico Los vocablos de la lengua inglesa de uso frecuente en las Áreas de la Administración, la Contaduría y la Informática Administrativa.

\section{Humanidades:}

En la UNAM se aprecia con interés el contenido de los seis (6) siguientes cursos:

-Primer Semestre: Estrategias de solución de problemas. Estrategias cognoscitivas para la solución de problemas; aplicación de dichas estrategias a casos prácticos.

\section{-Segundo Semestre: Dinámica Social y Teoría del Emprendedor Hechos y tendencias relevantes que se presenten en la sociedad contem- poránea, así como su efecto en las organizaciones; desarrollo de actitudes emprendedoras, de mejoramiento intelectual y de innovación.}


-Sexto Semestre: Investigación de Operaciones. Aplicación de modelos de investigación de operaciones a problemas contables.

-Octavo Semestre: Micro, Pequeña y Mediana Empresa. La importancia de la micro, pequeña y mediana empresa como generadoras de fuentes de trabajo y la conveniencia de crear su propia empresa para facilitar el desarrollo profesional.

-Noveno Semestre: Seminario de Tesis. Las características de una tesis profesional en el campo de la contaduría; las disposiciones reglamentarias sobre titulación y las líneas de investigación que en el campo de la contaduría se desarrollan en la facultad y en el país, para definir su tema de tesis.

-Décimo Semestre: Seminario de Tesis. Elaboración de su proyecto de tesis y avance en el desarrollo de su proyecto de tesis.

\section{CONCLUSIONES}

1. En las últimas reuniones profesionales de Contadores Públicos y de Docentes Universitarios del Área Contable, llevadas a cabo en el Perú y en otros países de América, se han planteado diversas ponencias sobre el futuro profesional del Contador Público en el Siglo XXI; se reclama un profundo cambio en el perfil del Contador Público, para convertirlo en un profesional de corte mundial o multinacional, con aptitudes y conocimientos necesarios para desarrollarse en un mundo globalizado, cada vez más competitivo. Por lo tanto, se impone la obligación de actualizar constantemente el Currículo o Plan de Estudios de las universidades que forman Contadores Públicos.

2. Forma parte de este importante proceso de actualización curricular, el desarrollo de las actividades de investigación universitaria, para alcanzar niveles de excelencia en la formación del Contador Público. Por lo tanto, se requiere implementar una planificación adecuada de programas de investigación, debidamente integrada con el temario curricular de los cursos de estudios de la carrera profesional y de la obtención de grados académicos y título profesional; así como en la capacitación de docentes e investigadores, que permitan multiplicar su número con visión estratégica, para responder a las exigencias del nuevo milenio.

3. En el Perú, se ha creado conciencia de que se necesita mejorar los vigentes programas curriculares de estudios universitarios, que aplican las facultades encargadas de formar Contadores Públicos. Esta tarea de cambio no es fácil por cuanto requiere de la ejecución de varias etapas operativas, como diagnóstico, evaluación, programación, realización y obtención de resultados. En el caso de la Facultad de 
Ciencias Contables de la UNMSM, desde 1996 ya se ha aplicado un nuevo Currículo con las características de modernidad y adelanto técnico que se menciona en el presente trabajo de investigación, con óptimos resultados.

4. Las propuestas curriculares que se plantean en este estudio, deben ser consideradas como una aportación inicial al proceso de actualización del Currículo o Plan de Estudios de la Facultad de Ciencias Contables de la UNMSM y de las demás universidades peruanas que forman Contadores Públicos y ser aplicadas en armonía con sus programas actuales de desarrollo curricular.

\section{BIBLIOGRAFIAA}

ASOCIACIÓN INTERAMERICANA DE CONTABILIDAD. En Revista InterAmérica (abril-junio 1998) - (julio- setiembre 1998) - (octubre-diciembre 1998).

INTERNET. Universidad Nacional Autónoma de México - Facultad de Contaduría y Administración - Licenciado en Contaduría - Plan de Estudios.

INTERNET. Universidad de Buenos Aires Argentina - Facultad de Ciencias Económicas - Contador Público - Plan de Estudios.

XXIII CONFERENCIA INTERAMERICANA DE CONTABILIDAD. Trabajos Interamericanos Puerto Rico - agosto 1999.

XXIII CONFERENCIA INTERAMERICANA DE CONTABILIDAD. Trabajos Técnicos Nacionales - Puerto Rico - agosto 1999.

ELIZONDOLÓPEZ, A.

Temática Contable sobre Actividades Académicas - Docencia, Investigación y aprendizaje.
UNIVERSIDAD AUTÓNOMA DE GUADALAJARA - MÉXICO. En Folleto: Contador Público.

UNIVERSIDAD CATÓLICA DE CÓRDOVA ARGENTINA. En Folleto: Contador Público. 